\title{
Psychotropic drug use before and during physical restraint for mechanically ventilated critically ill adults
}

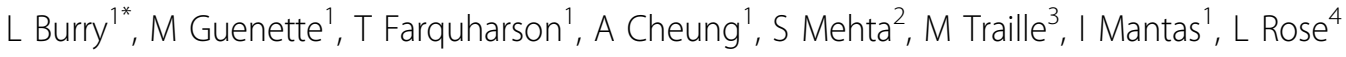 \\ From ESICM LIVES 2015 \\ Berlin, Germany. 3-7 October 2015
}

\section{Background}

Physical restraint (PR) is common in Canadian intensive care units (ICUs); used in up to $76 \%$ of mechanically ventilated patients. $\left[{ }^{1}\right]$ Psychotropic drugs may minimize PR used for agitation, promoting patient safety and preventing treatment interference. No data describes the psychotropic drug use around the time of PR application.

\section{Objective}

To characterize alterations to psychotropic drug regimens 1 hour prior and 6 hours after PR application for critically ill, mechanically ventilated adults.

\section{Methods}

Prospective single centre observational study (Feb 2014 to Jan 2015). Eligible patients were physically restrained while receiving mechanical ventilation. Psychotropic drug data were collected 1 hour before PR and for 6 hours after PR. We recorded PR indications, psychotropic drug interventions including new drug initiation, dose increase, dose decrease, and drug cessation. We documented total duration of PR, Sedation Agitation Scale (SAS) scores and presence of delirium (Intensive Care Delirium Screening Checklist score $\geq 4$ ). We used McNemar's tests to compare across time points.

\section{Results}

We enrolled 93 patients meeting our inclusion criteria. Mean age 59 years, $53 \%$ male, and $65 \%$ medical admissions. Median (IQR) mechanical ventilation duration was $5(2,9)$ days. 111 indications for PR included prevention of treatment interference $(90,81 \%)$, agitation $(13,12 \%)$, physical violence towards staff $(5,5 \%)$, and self-harm (3, 3\%). Median (IQR) duration of restraint application

Table 1

\begin{tabular}{llll}
\hline Drug therapy [n (\%)] $(\mathbf{N}=\mathbf{9 3})$ & pre-PR & post-PR & P \\
\hline Any psychotropic drug intervention & $52(56)$ & $80(86)$ & $<0.01$ \\
\hline a) New drug/increase b)Drug stop/decrease & a) $42(45)$ b) $10(11)$ & a) 69 (74) b) 11 (12) & a) $<0.01$ b) 1.00 \\
\hline No change & $23(25)$ & $7(8)$ & $<0.01$ \\
\hline No psychotropic drug & $18(19)$ & $6(6)$ & $<0.01$ \\
\hline Drug class (received $>1$ drug) & & $50(54)$ & $<0.01$ \\
\hline Opioids & $19(20)$ & $27(29)$ & 0.44 \\
\hline Non-benzodiazepine sedatives & $22(24)$ & $29(31)$ & 0.18 \\
\hline Benzodiazepines & $20(22)$ & $10(11)$ & 0.11 \\
\hline Antipsychotics & $4(4)$ &
\end{tabular}

${ }^{1}$ Mount Sinai Hospital, Pharmacy, Toronto, Canada

Full list of author information is available at the end of the article 
Table 2

\begin{tabular}{ll}
\hline Sedation $(\mathbf{N}=\mathbf{5 1})$ & $\mathbf{n}(\%)$ \\
\hline Appropriate pre/post (SAS 3-4) & $12(24)$ \\
\hline Achieved SAS 3-4 post & $8(16)$ \\
\hline Agitated pre/post (SAS 5-7) & $8(16)$ \\
\hline Oversedated pre/post (SAS 1-2) & $8(16)$ \\
\hline SAS 3-4 pre: SAS 5-7 post & $6(12)$ \\
\hline SAS 5-7 pre: SAS 1-2 post & $5(10)$ \\
\hline SAS 1-2 pre: SAS 5-7 post & $4(8)$ \\
\hline
\end{tabular}

was $21(9,70)$ hours. Of the 69 patients with delirium screening documented, 23 (33\%) were delirious. More patients received a psychotropic drug intervention after PR (52 vs $80, \mathrm{P}<0.001$ ) and more patients received an opioid after PR (19 vs $50, \mathrm{P}<0.001)$. Use of other drugs did not differ (Table 1). Sedation profile of the 51 patients with SAS scores documented pre and post is shown in Table 2. Adverse events during PR were uncommon with unintentional device removal in 7 (8\%) patients.

\section{Conclusions}

Psychotropic drug interventions were more common after PR application with opioids used most frequently suggesting priority of analgesia over sedation. Most patients had a SAS score indicating appropriate or oversedation in the hour prior suggesting PR was used more often as a preemptive approach.

\section{Authors' details}

${ }^{1}$ Mount Sinai Hospital, Pharmacy, Toronto, Canada. ${ }^{2}$ Mount Sinai Hospital, Medicine, Toronto, Canada. ${ }^{3}$ Mount Sinai Hospital, Nursing, Toronto, Canada. ${ }^{4}$ University of Toronto, Nursing, Toronto, Canada.

Published: 1 October 2015

\section{Reference}

1. Mehta, et al: JAMA 2012, 308:1985-92.

doi:10.1186/2197-425X-3-S1-A28

Cite this article as: Burry et al:: Psychotropic drug use before and

during physical restraint for mechanically ventilated critically ill adults. Intensive Care Medicine Experimental 2015 3(Suppl 1):A28.

\section{Submit your manuscript to a SpringerOpen ${ }^{\mathcal{D}}$ journal and benefit from:}

- Convenient online submission

- Rigorous peer review

- Immediate publication on acceptance

- Open access: articles freely available online

- High visibility within the field

- Retaining the copyright to your article

Submit your next manuscript at $>$ springeropen.com 